\title{
Applicability of Matrix Inverse in Simple Model of Economics - An Analysis
}

\author{
Mr. Anupam Sarma \\ Department of Economics M.N.C.Balika Mahavidyalaya Nalbari(Assam)-781335 Country-India
}

\section{Introduction:}

The knowledge of matrices is very important not only in mathematics, but also in economics, sociology and modern psychology including industrial management. It should be important to note that the evolution of concept of matrices is the result of an attempt to obtain compact and simple methods of solving a system of linear equations. Matrix algebra is also highly significant as it can enable us to do many things. It provides information regarding the testing of existence of a solution by evaluation of a determinant. While for solving a system of linear equations the role of matrix inverse method is highly effective.

Matrix inverse can be determined only when the given matrix is non-singular in nature. In simple sense we can say that matrix inverse can be determined only under the condition that determinant value of the given matrix is not become equal to zero. Matrix inverse, in application is defined as the ratio of adjoint matrix and determinant value of that matrix. The adjoint matrix is the transpose of cofactor matrix where cofactor of each and every element of the given matrix is determined on the basis of minor of the matrix. If $\mathrm{A}$ is assumed as any square matrix where $|A| \neq 0$ then matrix inverse usually denoted by $\mathrm{A}^{-1}$ is defined as -

$$
A^{-1}=\frac{\operatorname{Adj} . A}{|A|}
$$

We must have to follow certain important steps for using matrix inverse which are as follows.

- The determinant value of the given matrix should be found out which should not be zero, otherwise it cannot perfectly determine inverse of the matrix.

- The cofactors of each and every element of the coefficient matrix should be determined with their respective minors.

- The cofactor matrix should be found out which is the arrangement of respective rows and columns of determined cofactors.

- More importantly, the adjoint matrix which is the transpose of the cofactor matrix should be calculated.

- Finally, by using the formula given in equation (i) the inverse of matrix can be determined.

\section{Objectives:}

The major objectives of this paper are -

- To analyse the role of matrix inverse for solving linear market model.

- To analyse the effectiveness of inverse of matrix for determining equilibrium national income, consumption expenditure and taxes.

- Analysing the role of the same rule for solution of IS-LM model in economics.

- To study the role of matrix inverse under two goods market model.

\section{Methodology:}

The methodology used in this paper to study the above mentioned objectives is completely analytical and descriptive in nature. The models which are used in this paper are collected from indirect sources like from various books of mathematical economics.

\section{Discussions:}

In economics, matrix algebra is used dominantly in various purposes for determining the equilibrium values of a system of linear economic equations. The application of matrix inverse is very much significant in this regard which are discussed below.

\section{Solution of Linear Market Model:}

In linear market model, quantity demand $\left(\mathrm{Q}_{\mathrm{d}}\right)$ is a function of price $(\mathrm{P})$ of the product and quantity supply $\left(\mathrm{Q}_{\mathrm{s}}\right)$ is an increasing function of price $(\mathrm{P})$ where market clearing conditions prevail. We can construct the linear market model as - 
$\mathrm{Q}_{\mathrm{d}}=\mathrm{a}-\mathrm{bP}$

$\mathrm{a}, \mathrm{b}>0$

$\mathrm{Q}_{\mathrm{s}}=-\mathrm{c}+\mathrm{dP}$

$c, d>0$

$\mathrm{Q}_{\mathrm{d}}=\mathrm{Q}_{\mathrm{s}}$ (Market clearing condition)

The above given model can be arranged as follows:

$$
\left.\begin{array}{r}
Q_{d}+b P=a \\
Q_{s}-d P=-c \\
Q_{d}-Q_{s}=0
\end{array}\right\}----------------(i i)
$$

We can arrange equation (ii) in matrix form as -

$$
\begin{aligned}
& {\left[\begin{array}{rrr}
1 & 0 & b \\
0 & 1 & -d \\
1 & -1 & 0
\end{array}\right]\left[\begin{array}{l}
Q_{d} \\
Q_{s} \\
P
\end{array}\right]=\left[\begin{array}{l}
a \\
-c \\
0
\end{array}\right] } \\
\Rightarrow A & \times=B \\
\Rightarrow & \times=A^{-1} B-------(i i i)
\end{aligned}
$$

Now,

$$
\begin{aligned}
& |A|=\left|\begin{array}{rrr}
1 & 0 & b \\
0 & 1 & -d \\
1 & -1 & 0
\end{array}\right| \quad=1(0-d)-0+b(0-1) \\
& \because|A| \neq 0, \quad \therefore A^{-1} \text { exists. }
\end{aligned}
$$

Now, cofactor matrix of $\mathrm{A}$ is -

$$
\begin{aligned}
& {\left[\begin{array}{lllll}
(-1)^{1+1} & (0-d) & (-1)^{1+2}-(0+d) & (-1)^{1+3} & (0-1) \\
(-1)^{2+1}-(0+b) & (-1)^{2+2} & (0-b) & (-1)^{1+3}-(-1-0) \\
(-1)^{3+1} & (0-b) & (-1)^{3+2}-(-d-0) & (-1)^{3+3} & (1-0)
\end{array}\right]} \\
& =\left[\begin{array}{ccc}
-d & -d & -1 \\
-b & -b & 1 \\
-b & d & 1
\end{array}\right]
\end{aligned}
$$

$\therefore$ Adj. $A=$ Transpose of cofactor matrix A

$$
=\left[\begin{array}{ccc}
-d & -b & -b \\
-d & -b & d \\
-1 & 1 & 1
\end{array}\right]
$$

Now, inverse of matrix $\mathrm{A}$ is given by

$$
\begin{aligned}
A^{-1} & =\frac{\operatorname{Adj} . A}{|A|} \\
& =\frac{\left[\begin{array}{rrr}
-d & -b & -b \\
-d & -b & d \\
-1 & 1 & 1
\end{array}\right]}{-(b+d)}
\end{aligned}
$$


By substituting $\mathrm{A}^{-1}$ in equation (iii), we have

$$
\begin{aligned}
\mathrm{X} & =\frac{\left[\begin{array}{ccr}
-d & -b & -b \\
-d & -b & d \\
-1 & 1 & 1
\end{array}\right]}{-(b+d)}\left[\begin{array}{l}
a \\
-c \\
0
\end{array}\right] \\
\Rightarrow\left[\begin{array}{l}
Q_{d} \\
Q_{s} \\
P
\end{array}\right] & =\left[\begin{array}{l}
\frac{-a d+b c-0}{-(b+d)} \\
\frac{-a d+b c-0}{-(b+d)} \\
\frac{-a-c+0}{-(b+d)}
\end{array}\right]
\end{aligned}
$$

$\therefore$ The equilibrium solution is

$$
\begin{aligned}
& \bar{Q}_{d}=\frac{-a d+b c}{-(b+d)}=\frac{a d-b c}{b+d} \\
& \bar{Q}_{s}=\frac{-a d+b c}{-(b+d)}=\frac{a d-b c}{b+d} \\
& \bar{P}=\frac{-a-c}{-(b+d)}=\frac{a+c}{b+d}
\end{aligned}
$$

\section{Solution of Simple National Income Model:}

In economics, the simple national income model is given by

$$
\begin{array}{ll}
Y=C+I_{0}+G_{0} & \\
C=a+b(Y-T) & a>0, \quad 0<b<1 \\
T=t Y & 0<t<1
\end{array}
$$

Where $\mathrm{Y}$ is national income, $\mathrm{C}$ is consumption expenditure, $\mathrm{T}$ is tax, $\mathrm{t}$ is rate of tax, $\mathrm{I}_{0}$ is government investment and $\mathrm{G}_{0}$ is government expenditure.

The above given equations can be arranged as

$$
\begin{aligned}
& Y-C=I_{0}+G_{0}----------(i v) \\
& -b Y+C+b T=a---------(v) \\
& -t Y+T=0------------(v i)
\end{aligned}
$$

When we arrange equations (iv), (v) and (vi) in matrix form, we have

$$
\begin{aligned}
& {\left[\begin{array}{rrr}
1 & -1 & 0 \\
-b & 1 & b \\
-t & 0 & 1
\end{array}\right]\left[\begin{array}{l}
Y \\
C \\
T
\end{array}\right]=\left[\begin{array}{c}
I_{0}+G_{0} \\
a \\
0
\end{array}\right]} \\
& \Rightarrow A \quad \times \quad=B \\
& \Rightarrow \quad \times \quad=A^{-1} B-------(v i)
\end{aligned}
$$

Now, 


$$
\begin{aligned}
|A| & =\left|\begin{array}{rrr}
-1 & -1 & 0 \\
-b & 1 & b \\
-t & 0 & 1
\end{array}\right| \\
& =1(1-0)+1(-b+b t)+0 \\
& =1-b+b t
\end{aligned}
$$

$\because 0<\mathrm{b}<1$ and $0<\mathrm{t}<1$, therefore $|A| \neq 0$ and hence $A^{-1}$ exits.

Now, the cofactor matrix of $\mathrm{A}$ is

$\therefore$ Adj. $A=$ Transpose cofactor matrix A

$$
\begin{aligned}
& {\left[\begin{array}{lllll}
(-1)^{2} & (1-0) & (-1)^{3}-(b+b t) & (-1)^{4} & (-0+t) \\
(-1)^{3}-(-1-0) & (-1)^{4} & (1+0) & (-1)^{5}-(0-t) \\
(-1)^{4} & (-b-0) & (-1)^{5}-(b+0) & (-1)^{6} & (1-b)
\end{array}\right]} \\
& =\left[\begin{array}{llc}
1 & b-b t & t \\
1 & 1 & t \\
-b & -b & 1-b
\end{array}\right]
\end{aligned}
$$

$$
=\left[\begin{array}{ccr}
1 & 1 & -b \\
b-b t & 1 & -b \\
t & t & 1-b
\end{array}\right]
$$

Now, inverse of matrix $\mathrm{A}$ is given as

$$
\begin{aligned}
A^{-1} & =\frac{A d j . A}{|A|} \\
& =\frac{\left[\begin{array}{ccr}
1 & 1 & -b \\
b-b t & 1 & -b \\
t & t & 1-b
\end{array}\right]}{1-b+b t}
\end{aligned}
$$

By substituting $A^{-1}$ in equation (vii) we have

$$
\begin{gathered}
X=\frac{\left[\begin{array}{ccr}
1 & 1 & -b \\
b-b t & 1 & -b \\
t & t & 1-b
\end{array}\right]}{1-b+b t}\left[\begin{array}{c}
I_{0}+G_{0} \\
a \\
0
\end{array}\right] \\
\Rightarrow\left[\begin{array}{l}
Y \\
C \\
T
\end{array}\right]=\left[\begin{array}{l}
\frac{\left(I_{0}+G_{0}\right)+a-0}{1-b+b t} \\
\frac{(b-b t)\left(I_{0}+G_{0}\right)+a-0}{1-b+b t} \\
\frac{t\left(I_{0}+G_{0}\right)+t \cdot a+0}{1-b+b t}
\end{array}\right]
\end{gathered}
$$


$\therefore$ The solution of the model is

$$
\begin{aligned}
& \bar{Y}=\frac{I_{0}+G_{0}+a}{1-b+b t} \\
& \bar{C}=\frac{\left(I_{0}+G_{0}\right)(b-b t)+a}{1-b+b t} \\
& \bar{T}=\frac{\left(I_{0}+G_{0}+a\right) t}{1-b+b t}
\end{aligned}
$$

\section{General Solution of IS-LM Model:}

The IS-LM model is related to determination of equilibrium rate of interest( $\mathrm{r}$ ) and income (Y) where product market (IS) and money market (LM) simultaneously achieves equilibrium. The linear equation related to product market is given as

$Y=C+I$

Where

$$
C=a+b Y \quad a>0 \text { and } 0<b<1
$$

$$
I=\alpha+\beta r \quad \alpha>0 \text { and } \beta<0
$$

Again, the mathematical form of LM model is given as

$$
M_{d}=M_{s}
$$

Where,

$$
\begin{array}{ll}
M_{d}=\gamma+\delta Y+\lambda r & \gamma, \delta>0 \text { and } \lambda<0 \\
M_{s}=K & K \text { is fixed moneysup ply }>0
\end{array}
$$

Here, $\mathrm{M}_{\mathrm{d}}$ and $\mathrm{M}_{\mathrm{s}}$ are respectively money demand and money supply.

We can arrange the IS-LM model as

$$
\begin{aligned}
& Y=C+I \\
\Rightarrow & Y=a+b Y+\alpha+\beta r \\
\Rightarrow & Y-b Y-\beta r=a+\alpha \\
\Rightarrow & (1-b) Y-\beta r=a+\alpha
\end{aligned}
$$

Again, $\mathrm{M}_{\mathrm{d}}=\mathrm{M}_{\mathrm{s}}$

$$
\begin{aligned}
& \Rightarrow \gamma+\delta Y+\lambda r=K \\
& \Rightarrow \delta Y+\lambda r \quad=K-\gamma
\end{aligned}
$$

When we arrange equations (viii) and (ix) in matrix form, we have

$$
\begin{aligned}
& {\left[\begin{array}{lr}
1-b & -\beta \\
\delta & \lambda
\end{array}\right]\left[\begin{array}{l}
Y \\
r
\end{array}\right]=\left[\begin{array}{l}
a+\alpha \\
K-\gamma
\end{array}\right]} \\
& \Rightarrow A . X=B \\
& \Rightarrow X=A^{-1} B---------
\end{aligned}
$$

Now,

$$
\begin{aligned}
& |A|=\left|\begin{array}{lr}
1-b & -\beta \\
\delta & \lambda
\end{array}\right| \\
& =\lambda(1-b)+\beta \delta \\
& =\lambda-\lambda b+\beta \delta
\end{aligned}
$$




$$
\because|A| \neq 0, \quad \therefore A^{-1} \text { exists }
$$

Now, cofactor matrix of A

$$
\begin{aligned}
& {\left[\begin{array}{ll}
(-1)^{2} \lambda & (-1)^{3} \delta \\
(-1)^{3}(-\beta) & (-1)^{4}(1-b)
\end{array}\right] } \\
= & {\left[\begin{array}{ll}
\lambda & -\delta \\
\beta & 1-b
\end{array}\right] }
\end{aligned}
$$

$\therefore \operatorname{Adj} . A=$ Transpose of cofactor matrix A

$$
=\left[\begin{array}{ll}
\lambda & \beta \\
-\delta & 1-b
\end{array}\right]
$$

Now, inverse of matrix $\mathrm{A}$ is given as

$$
\begin{aligned}
A^{-1} & =\frac{\operatorname{Adj} . A}{|A|} \\
& =\frac{\left[\begin{array}{rr}
\lambda & \beta \\
-\delta & 1-b
\end{array}\right]}{\lambda-\lambda b+\beta \delta}
\end{aligned}
$$

When we substitute $A^{-1}$ in equation (x), we have

$$
\begin{aligned}
X & \left.=\frac{\left[\begin{array}{cc}
\lambda & \beta \\
-\delta & 1-b
\end{array}\right]\left[\begin{array}{l}
a+\alpha \\
\lambda-\lambda b+\beta \delta
\end{array}\right]}{K-\gamma}\right] \\
\Rightarrow\left[\begin{array}{l}
Y \\
r
\end{array}\right] & =\left[\begin{array}{l}
\frac{\lambda(a+\alpha)+\beta(K-\delta)}{\lambda-\lambda b+\beta \delta} \\
\frac{-\delta(a+\alpha)+(1-b)(K-\delta)}{\lambda-\lambda b+\beta \delta}
\end{array}\right]
\end{aligned}
$$

$\therefore$ The solution is

$$
\begin{aligned}
& \bar{Y}=\frac{(a+\alpha) \lambda+(K-\delta) \beta}{\lambda-\lambda b+\beta \delta} \\
& \bar{r}=\frac{(1-b)(K-\gamma)-\delta(a+\alpha)}{\lambda-\lambda b+\beta \delta}
\end{aligned}
$$

\section{Solution under Two Goods Market Model:}

The two goods market model is related to two markets producing two commodities which are sold in each respective market. In such a market, the matrix inverse is significant for determining the selling prices of commodities at equilibrium in each market. The derivation form of such a model is shown as follows.

In market 1 ,

$$
\begin{aligned}
& Q_{d 1}=a-b P_{1}+m P_{2} \quad a, b, m>0 \\
& Q_{s 1}=-c+d P_{1} \quad c, d>0 \\
& Q_{d 1}=Q_{s 1}(\text { Marketclearing condition) }
\end{aligned}
$$


Where $\mathrm{Q}_{\mathrm{d} 1}$ and $\mathrm{Q}_{\mathrm{s} 1}$ are respectively the quantity demand and supply in market $1, \mathrm{P}_{1}$ and $\mathrm{P}_{2}$ are the prices of market 1 and 2 respectively.

Again, in market 2

$Q_{d 2}=\alpha-\beta P_{1}+n P_{1} \quad \alpha, \beta, n>0$

$Q_{s 2}=-\gamma+\lambda P_{2} \quad \gamma, \lambda>0$

$Q_{d 2}=Q_{s 2}$ (Market clearing condition)

We can arrange the equations in both markets as,

In market 1 ,

In market 2 ,

$$
\begin{aligned}
& Q_{d 1}=Q_{S 1} \\
& \Rightarrow a-b P_{1}+m P_{2}=-c+d P_{1} \\
& \Rightarrow a-c=b P_{1}+d P_{1}-m P_{2} \\
& \Rightarrow(b+d) P_{1}+m P_{2}=a+c-----------(x i)
\end{aligned}
$$

$$
\begin{aligned}
& Q_{d 2}=Q_{S 2} \\
& \Rightarrow \alpha-\beta P_{2}+n P_{1}=-\gamma+\lambda P_{2} \\
& \Rightarrow \alpha+\gamma=-n P_{1}+\lambda P_{2}+\beta P_{2} \\
& \Rightarrow-n P_{1}+(\lambda+\beta) P_{2}=\alpha+\gamma-----------(x i i)
\end{aligned}
$$

When we arrange equations (xi) and (xii) in matrix form, we have

$$
\begin{aligned}
& {\left[\begin{array}{cc}
b+d & -m \\
-n & \lambda+\beta
\end{array}\right]\left[\begin{array}{l}
P_{1} \\
P_{2}
\end{array}\right]=\left[\begin{array}{l}
a+c \\
\alpha+\gamma
\end{array}\right]} \\
& \Rightarrow A \quad \times=B \\
& \Rightarrow \quad \times=A^{-1} B-----------(\text { xiii })
\end{aligned}
$$

Now,

$$
\begin{aligned}
&|A|=\left|\begin{array}{cc}
b+d & -m \\
-n & \lambda+\beta
\end{array}\right|=(b+d)(\lambda+\beta)-n m \\
& \because|A| \neq 0, \therefore A^{-1} \text { exists }
\end{aligned}
$$

Now, cofactor matrix of $\mathrm{A}$ is$$
\left[\begin{array}{lll}
(-1)^{2}(\lambda+\beta) & (-1)^{3} & (-n) \\
(-1)^{3}(-m) & (-1)^{4} & (b+d)
\end{array}\right]
$$$$
=\left[\begin{array}{ll}
(\lambda+\beta) & n \\
m & (b+d)
\end{array}\right]
$$

$\therefore A d j . A=$ Transpose of cofactor matrix A

$$
=\left[\begin{array}{cc}
(\lambda+\beta) & m \\
n & (b+d)
\end{array}\right]
$$

Now, inverse of matrix A is 


$$
\begin{aligned}
A^{-1} & =\frac{\operatorname{Adj} . A}{|A|} \\
& =\frac{\left[\begin{array}{cc}
(\lambda+\beta) & m \\
n & (b+d)
\end{array}\right]}{(b+d)(\lambda+\beta)-n m}
\end{aligned}
$$

When we substitute $A^{-1}$ in equation (xiii), we have

$$
\begin{aligned}
& X=\frac{\left[\begin{array}{cc}
(\lambda+\beta) & m \\
n & (b+d)
\end{array}\right]}{(b+d)(\lambda+\beta)-n m} \cdot\left[\begin{array}{l}
a+c \\
\alpha+\gamma
\end{array}\right] \\
& \Rightarrow\left[\begin{array}{l}
P_{1} \\
P_{2}
\end{array}\right]=\left[\begin{array}{l}
\frac{(\lambda+\beta)(a+c)+m(\alpha+\gamma)}{(b+d)(\lambda+\beta)-n m} \\
\frac{n(a+c)+(b+d)(\alpha+\gamma)}{(b+d)(\lambda+\beta)-n m}
\end{array}\right] \\
& \therefore \overline{P_{2}}=\frac{(\lambda+\beta)(a+c)+m(\alpha+\gamma)}{(b+d)(\lambda+\beta)-n m} \\
& \therefore \overline{P_{2}}=\frac{n(a+c)+(b+d)(\alpha+\gamma)}{(b+d)(\lambda+\beta)-n m}
\end{aligned}
$$

These are the equilibrium prices of commodities in each of the market.

\section{Conclusion:}

The concept of matrix inverse is very useful in economics in solving simultaneous equations, in inputoutput analysis and even in regression analysis. While for determining the sectoral outputs in static and dynamic input-output analysis, the application of matrix inverse is very important. From our discussion, it is clear that inversion of matrix is possible if and only if two conditions are satisfied. Firstly, matrix whose inverse is required is a square matrix otherwise we cannot be able to form the determinant of the matrix. Secondly, the determinant of the matrix should not be zero which implies that the matrix whose inverse is required should be non-singular.

\section{References:}

[1]. R.G.D Allen, Mathematical Analysis for Economics, A.I.T.B.S. Publishers (2008)

[2]. Srinath Baruah, Basic Mathematics \& its applications in Economics, Macmillan (2011).

[3]. A.C. Chiang, Fundamental Methods of Mathematical Economics, Mcgraw-Hill International Editions (1984)

[4]. E.T. Dowling, Introduction to Mathematical Economics, Mcgraw-Hill (2001)

[5]. R.K.Lekhi \& J. Singh, Agricultural Economics: An Indian Perspective, Kalyani Publishers, Ludhiana (2010).

[6]. Mehta- Madnani, Mathematics for Economists, Sultan Chand \& Sons (2007).

[7]. Anupam Sarma, Mathematical Economics \& Its Applications, Kasturi Printers \& Publishers, Guwahati (2014)

[8]. R.N. Soni, Leading Issues in Agricultural Economics, Vishal Publishing Co., Delhi. 